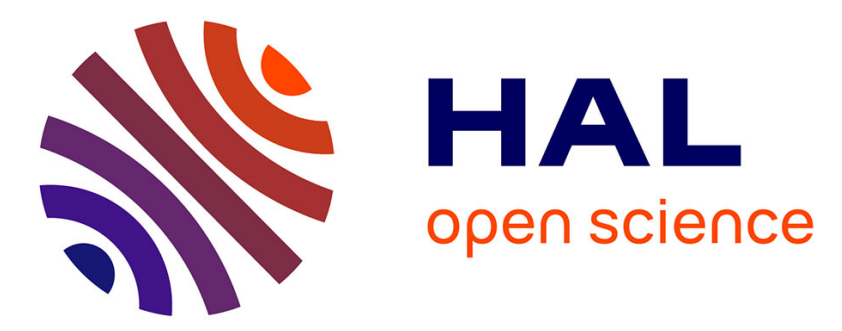

\title{
Design of Electrically Small 3D Wire Antennas for UHF RFID applications Using Genetic Algorithm
}

\author{
Fateh Benmahmoud, Pierre Lemaitre-Auger, Smail Tedjini
}

\section{To cite this version:}

Fateh Benmahmoud, Pierre Lemaitre-Auger, Smail Tedjini. Design of Electrically Small 3D Wire Antennas for UHF RFID applications Using Genetic Algorithm. 2017 XXXIInd General Assembly and Scientific Symposium of the International Union of Radio Science (URSI GASS), Aug 2017, Montreal, Canada. pp.1-4, 10.23919/ursigass.2017.8105332 . hal-02388141

HAL Id: hal-02388141

https://hal.univ-grenoble-alpes.fr/hal-02388141

Submitted on 1 Dec 2019

HAL is a multi-disciplinary open access archive for the deposit and dissemination of scientific research documents, whether they are published or not. The documents may come from teaching and research institutions in France or abroad, or from public or private research centers.
L'archive ouverte pluridisciplinaire HAL, est destinée au dépôt et à la diffusion de documents scientifiques de niveau recherche, publiés ou non, émanant des établissements d'enseignement et de recherche français ou étrangers, des laboratoires publics ou privés. 


\title{
Design of Electrically Small 3D Wire Antennas for UHF RFID applications Using Genetic Algorithm
}

\author{
Fateh Benmahmoud, Pierre Lemaitre-Auger, and Smail Tedjini \\ Grenoble Alpes University - LCIS, 26902 Valence, FRANCE, http://lis.grenoble-inp.fr
}

\begin{abstract}
In this work, we discuss the design of electrically small antennas for UHF RFID tags. We implemented a genetic algorithm to design crooked-wire antennas that occupy a relatively compact size as small as $0.08 \lambda$, while exhibiting high efficiency and an acceptable radiation resistance value suitable for matching RFID chips.
\end{abstract}

Index Terms - RFID, Electrically small antennas, genetic algorithms (GA), wire antennas, UHF-tag, optimization.

\section{Introduction}

Rising interest and huge growth of the radio-frequency identification (RFID) technology happened in the last decade and similar to the evolution of several technologies, RFID devices tends to be more and more miniature, which implies the necessity of using very compact RFID tags that must exhibit high performance. Since RFID chips are very small, the miniaturization of an RFID tag means the miniaturization its antenna. This requires a long optimization process that rapidly becomes a complex task if someone employs usual design techniques [1-2]. An antenna is said electrically small if its geometrical dimensions are much smaller than the wavelength of the radiated electromagnetic field [3]. In a more explicit way, Wheeler [4] defined an electrically small antenna as the antenna that satisfies the relation:

$$
k a<1
$$

$k=2 \pi \lambda, \lambda$ is the wavelength and $a$ the radius of sphere circumscribing the antenna. In the present study we consider that $a$ is the radius of the sphere that enclose the radiated part of antenna without the ground plane (Fig. 1).

Since the downsizing of an antenna affects significantly its performances such the radiation efficiency and the radiation resistance, a trade-off between these factors is required. The majority of the achieved works in the miniaturization of 3D wire antennas target the VHF band, and focus on the gain and bandwidth of the antenna [5-8]. In most cases, the obtained results were very encouraging and lead to achieve the targeted objectives.

In the same time, several numerical optimization techniques like genetic algorithm (GA) or particle swarm optimization (PSO) has proven their usefulness in the design of antennas in complex environments [9-11]. In this work, we report on the use of a (GA) in the optimization of the design of electrically small 3D wire antennas for UHF-RFID application. We especially focus on, antenna size and radiation efficiency.

In this investigation, in section 2, we use a multi-objective GA implemented on Matlab in conjunction with CST Microwave Studio (CST MWS) to search for resonant 3D wire structure that occupy the smallest volume, have high efficiency near the frequency $868 \mathrm{MHz}$, and present an acceptable radiation resistance. In section 3 , we investigate two topologies of crooked-wire antennas, and discuss their performances. We also use the technique described in [12] to increase the radiation resistance and achieve required matching.

\section{Approach}

The crooked wire antenna optimized in this work with the help GA consists in a determined number of wires connected in series in free space. Each antenna have a radius $R=0.8 \mathrm{~mm}$ and a linear length between $0.2 \lambda$ and $0.4 \lambda$. We consider that the structure is enclosed inside a sphere of radius $a$, that circumscribes the whole antenna, over an infinite ground plane as it is shown in Figure 1.

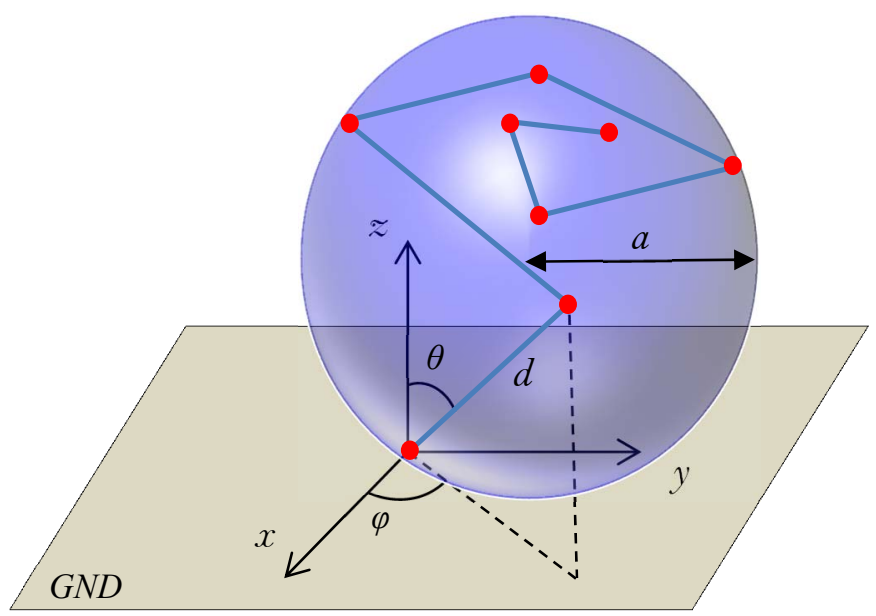

Figure 1. Shematic representation of a crooked wire antenna having 7 wires.

Two different topologies of antennas are studied: 7 wires and 10 wires. Each wire is defined by three parameters: elevation angle $\theta$, azimuth angle $\varphi$ and its length $d$. 
The values of the elevation angles are fixed between $0^{\circ}$ and $180^{\circ}$ with only 32 allowed values (at every $5.80^{\circ}$ ), except the first wire angle which is limited to $\left[0^{\circ}-90^{\circ}\right]$ with 16 allowed values (at every $5.80^{\circ}$ ). The azimuth angles are between $0^{\circ}$ and $360^{\circ}$ with also 32 allowed values (at every $11.25^{\circ}$ ). Wires lengths between $0.02 \lambda$ and $0.04 \lambda$ for the 10 wires antenna and $0.03 \lambda$ to $0.06 \lambda$ for the 7 wires antenna, with 32 allowed values in both cases. Based on this parameterization, we code the 7 wires antenna with 21 genes and the 10 wires antenna with 30 genes.

The implemented GA is based on that proposed in [10] where the best half of every generation is selected for the production of the next generation. The GA starts by selecting randomly a population of $N$ chromosomes (i.e. $N$ antennas) and evaluate the performances of each one. In the present study, different population sizes were used because a too small population size could lead to poor solutions while a large population size increases the time needed to find a solution [13-15]. We used the technique based on the bias measure suggested by Grefenstette in [16] to test the initial population diversity over the entire search space. This limits the population size effect. We also added a geometrical filter to eliminate impossible configurations: intersections of wires, crossing the ground plane or very close parallel wires. All these elements are incorporated during the execution of GA as constraints to ensure a good reliability of the results.

In addition to the integrated constraint of diversity, we tried to reduce the probability of local minima convergence by making sure that the geometrically rejected chromosomes are replaced, at every iteration, by new ones randomly created and geometrically correct. This way of doing increases the performances of the GA and enhances diversity [17].

The present work is a multi-objective problem. Three costs functions were defined to achieve the desired goal:

Table 1. The cost functions with weights.

\begin{tabular}{|c|c|c|}
\cline { 2 - 3 } \multicolumn{1}{c|}{} & Cost function & Weight \\
\hline Cost 1 & Antenna size $(\mathrm{ka})$ & 0.8 \\
\hline Cost2 & $1-\eta$ & 0.1 \\
\hline Cost3 & $R_{\text {ref }}-R_{\text {rad }}$ & 0.1 \\
\hline
\end{tabular}

where $k=2 \pi / \lambda$ is the wave number, $\eta$ is the radiation efficiency, $R_{\text {rad }}$ is the radiation resistance and $R_{\text {ref }}$ is a reference to evaluate the radiation resistance. It is fixed here to $14 \Omega$ which is close to real part of the impedance of an RFID chip. Antenna size is calculated using Matlab while the radiation efficiency and the radiation resistance computed through numerical simulation with CST microwave studio. After simulation, a figure of merit calculated by the sum of the three costs with an appropriate weighing to predict the fitness of the simulated antenna (see Table 1).

\section{Results}

Processing of the GA for the 7 wires antenna took 45 iterations with an initial population of 32 chromosomes. For the 10 wires antenna with an initial population of 40 chromosomes 21 iterations were done. For both configurations, highly random shape were selected for the $3 \mathrm{D}$ antennas.

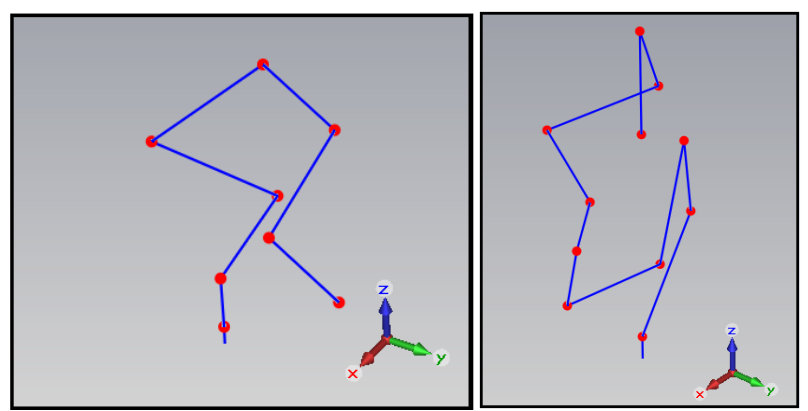

Figure 2. Designs of the obtained antennas: a) 7 wires antenna. b) 10 wires antenna.

The obtained antennas have a very small size compared to quarter-wave monopole, with a significant size reduction of $68 \%$ and $71 \%$ for the 7 wires antenna and the 10 wires antenna respectively; their total lengths are $0.243 \lambda$ and $0.285 \lambda$, with a value of $k a$ equal to 0.224 and 0.220 .

The radiation efficiencies of the 7 wires and 10 wires antenna are $91.47 \%$ and $96.0 \%$ respectively. Those results are good ones, especially when considering the size reduction. Both antennas have a near hemispherical coverage with a gain of $6.24 \mathrm{dBi}$ for the 7 wires antenna and $5.63 \mathrm{dBi}$ for the 10 wires antenna as shown in Fig. 3a and Fig. $3 b$.

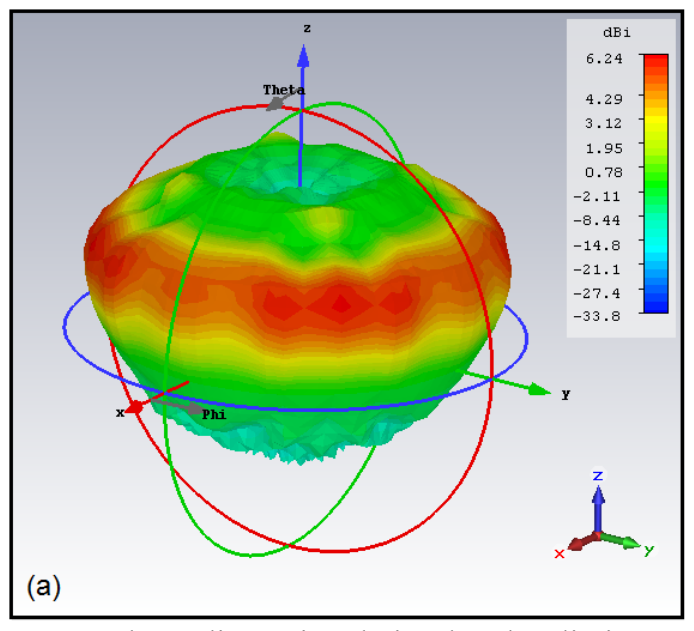

Figure 3a. Three-dimensional simulated radiation pattern of the obtained genetic antennas at $f=868 \mathrm{MHz}$ : 7 wires antenna 


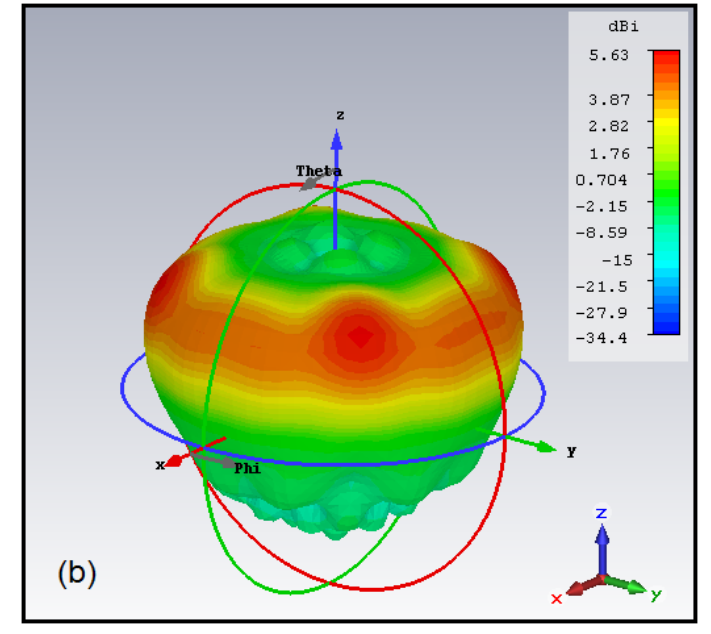

Figure 3b. Three-dimensional simulated radiation pattern of the obtained genetic antennas at $f=868 \mathrm{MHz}$ : 10 wires antenna.

The radiation resistance of the optimized antennas are very small: $5.41 \Omega$ and $4.47 \Omega$ respectively. The use of a matching technique is necessary to guarantee the functionality of the antennas. Among several matching techniques of wire antennas, we used the impedance adaptation technique described by S.Best in [12] to increase the radiation resistance and improve the impedance matching. This technique consists in using a parallel matching stub placed near the input of the antenna as it is shown in figure 4. The usual way to design a stub consists of calculating its length and its location from the antenna feeding. This method leads to large size of stub and increase the occupied volume of the antenna. To overcome this problem we try to find a small stub that increase the radiation resistance to a reasonable value, which is done after several simulations.

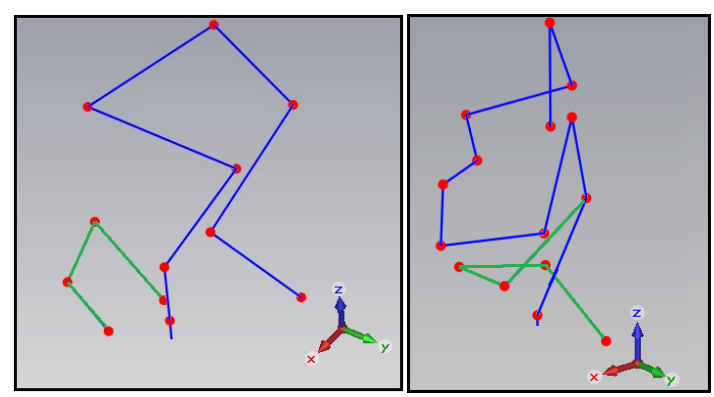

Figure 4. Designs of the obtained antennas with its matching stub: a) 7 wires antenna. b) 10 wires antenna.

With that technique, the radiation resistance of the antenna increased significantly, good matching with several UHF RFID chips is now possible. It worth mentioning that radiation efficiencies after matching was reduced to $85.61 \%$ and $95 \%$ which remains a satisfactory value, while the gain remains almost the same, its also important to mention that the size of the matched antennas was slightly larger. Numerical values obtained are summarized in Table 2.
Table 2. Impedances efficiencies and gain after impedance matching at $f=868 \mathrm{MHz}$.

\begin{tabular}{|c|c|c|}
\cline { 2 - 3 } \multicolumn{1}{c|}{} & 7 wires & 10 wires \\
\hline Impedance $(\Omega)$ & $13.9+\mathrm{j} 126.24$ & $24+\mathrm{j} 220.63$ \\
\hline Rad. Efficiency $(\%)$ & 85.61 & 95 \\
\hline Gain $(\mathrm{dBi})$ & 6.19 & 5.6 \\
\hline
\end{tabular}

\section{Conclusion}

We have shown that using GA small size wire antennas for RFID applications can be obtained with GA. The designed antennas have a good performance except for the radiation resistance. This is significantly improved with the use of a matching stub that increases the radiation resistance. There for, obtained antennas can be matched with RFID chips. They are very good candidate for UHF RFID tags for which size is an important matter. More results will be presented at the conference.

\section{References}

1. Gianvittorio, J.p., and Y. Rahmat-Samii, "Fractal antennas: a novel antenna miniaturization technique, and applications," IEEE Antennas and Propagation Magazine 44, 1, February 2002, pp. 20-36, doi:10.1109/74.997888.

2. Skrivervik, A.k., J.-F. Zurcher, O. Staub, and J.r. Mosig, "PCS antenna design: the challenge of miniaturization," IEEE Antennas and Propagation Magazine 43, 4, August 2001, pp. 12-27, doi:10.1109/74.951556.

3. Chu, L. J, "Physical Limitations of Omni - Directional Antennas," Journal of Applied Physics 19, 12, December 1948, pp. 1163-1175, doi:10.1063/1.1715038.

4. H.A. Wheeler, "Fundamental Limits of Small Antennas," Proceeding of the I.R.E. (IEEE), December 1947, pp. 14791484.doi:10.1109/jrproc.1947.226199.

5. Altshuler, E.e, "Electrically small self-resonant wire antennas optimized using a genetic algorithm," IEEE Transactions on Antennas and Propagation 50, 3, March 2002, pp. 297-300, doi:10.1109/8.999619.

6. Hornby, Gregory, Al Globus, Derek Linden, and Jason Lohn, "Automated Antenna Design with Evolutionary Algorithms," Space 2006, doi:10.2514/6.2006-7242.

7. Altshuler, E.e., and D.s. Linden, "Wire-antenna designs using genetic algorithms," IEEE Antennas and Propagation Magazine 39, 2, April 1997, pp. 33-43, doi:10.1109/74.584498.

8. Choo, Hosung, R.1. Rogers, and Hao Ling, "Design of electrically small wire antennas using a pareto genetic algorithm," IEEE Trans. on Antennas and Propagation 53, 3, March 2005, pp. 1038-1046, doi:10.1109/tap.2004.842404.

9. Robinson, J., and Y. Rahmat-Samii, "Particle Swarm Optimization in Electromagnetics," IEEE Transactions on Antennas and Propagation 52, 2, February 2004, pp. 397-407, doi:10.1109/tap.2004.823969. 
10. Rahmat-Samii, Yahya, \& Eric Michielssen, Electromagnetic optimization by genetic algorithms. New York: J. Wiley, 1999.

11. Siragusa, R., E. Perret, H. V. Nguyen, P. Lemaître-Auger, S. Tedjini, and C. Caloz, "Control of the Sensitivity of CRLH Interdigital Microstrip Balanced Structures Using a Co-Design Genetic Algorithm Approach," Applied Physics A 103, 3, January 2011, pp. 709-714. doi:10.1007/s00339-010-6205-z.

12. Best, S.r, "A discussion on the quality factor of impedance matched electrically small wire antennas," IEEE Transactions on Antennas and Propagation 53, 1, January 2005, pp. 502-508, doi:10.1109/tap.2004.837107.

13. M. Pelikan, D. E. Goldberg, and E. Cantú-Paz, "Bayesian optimization algorithm, population sizing, and time to convergence," Illinois Genetic Algorithms Laboratory, University of Illinois, Tech. Rep, 2000

14. Koumousis, V.k., and C.p. Katsaras, "A saw-tooth genetic algorithm combining the effects of variable population size and reinitialization to enhance performance," IEEE Transactions on Evolutionary Computation 10, 1, February 2006, pp. 19-28, doi:10.1109/tevc.2005.860765.

15. Yu, Tian-Li, Kumara Sastry, David E. Goldberg, and Martin Pelikan, "Population sizing for entropy-based model building in discrete estimation of distribution algorithms," Proceedings of the 9th annual conference on Genetic and evolutionary computation, 2007, doi:10.1145/1276958.1277080.

16. Bäck, Thomas, Evolutionary algorithms in theory and practice: evolution strategies, evolutionary programming, genetic algorithms, New York: Oxford University Press, 1996.

17. Pedro A. Diaz-Gomez and Dean F. Hougen, "Initial Population for Genetic Algorithms: A Metric Approach," proceedings of the International Conference on Genetic and Evolutionary Methods, 2007, pp. 25-28. 